Revista Colombiana de Obstetricia y Ginecología Vol. 66 No. 2 • Abril-Junio $2015 \bullet(131-137)$

\title{
HEMATOMA RETROPERITONEAL POSTERIOR A HISTERECTOMÍA VAGINAL CON COLPORRAFIA ANTERIOR Y POSTERIOR EN BOGOTÁ (COLOMBIA): REPORTE DE UN CASO Y REVISIÓN DE LA LITERATURA
}

\section{Retroperitoneal haematoma following vaginal hysterectomy with anterior and posterior vaginal wall repair in Bogotá, Colombia: Case report and review of the literature}

\section{Marcos Fidel Castillo-Zamora, $M D^{1}$; Julie A. Mora-Enríquez, $M^{2}$;} Fernando J. Monsalvo-Díaz, $M D^{3}$

Recibido: junio 16/14 - Aceptado: mayo 25/15

\section{RESUMEN}

Objetivo: reportar el caso de una paciente con hematoma retroperitoneal posterior a cirugía de corrección de prolapso por vía vaginal, y realizar revisión de la literatura médica sobre la anatomía del espacio retroperitoneal, la etiología, el diagnóstico, manejo y pronóstico del hematoma retroperitoneal posoperatorio en ginecología.

Materiales y métodos: se presenta el caso de una paciente con prolapso vaginal estadio II, a quien se le practicó histerectomía vaginal con colporrafia anterior y posterior, quien presentó como complicación un hematoma retroperitoneal. La paciente fue intervenida en el Hospital Universitario de La Samaritana, hospital público de tercer nivel de complejidad, centro de referencia del departamento de

1 Especialista en Ginecología y Obstetricia, Universidad Javeriana, Bogotá (Colombia). Profesor Asistente, Universidad de La Sabana, Chía (Colombia).marcoscz@unisabana.edu.co

2 Residente de IV año Ginecología y Obstetricia, Universidad de La Sabana, Hospital Universitario de La Samaritana, Bogotá (Colombia).

3 Especialista en Ginecología y Obstetricia, Universidad de La Sabana, Hospital Universitario de la Samaritana, Bogotá (Colombia).

fernandomonsalvo@hotmail.com
Cundinamarca, ubicado en Bogotá (Colombia). Se realizó una búsqueda de literatura médica en las bases de dados Medline vía PubMed, Jstor y Lilacs, con terminología MeSH "hysterectomy vaginal” y "retroperitoneal hematoma”. La búsqueda se limitó a los idiomas inglés y español entre los años 1980 a 2015.

Resultados: se encontraron 15 artículos de los cuales 3 describen casos secundarios a procedimientos ginecológicos. La revisión final se conforma de: 4 revisiones de tema, 8 reportes de caso, 2 series de casos, 1 estudio observacional descriptivo.

Conclusión: el hematoma retroperitoneal es una rara entidad que requiere de un alto índice de sospecha clínica para su diagnóstico. Existen varias herramientas diagnósticas, siendo la tomografía computarizada la de mayor utilidad. El manejo debe individualizarse a cada caso. En paciente estable se puede realizar manejo conservador con éxito o intervenciones endovasculares. Sin embargo, en pacientes inestables hemodinámicamente, la laparotomía es la conducta más recomendada.

Palabras clave: retroperitoneal, hematoma, histerectomía vaginal, hemorragia. 


\section{ABSTRACT}

Objective: To report the case of a patient who developed a retroperitoneal haematoma following prolapse correction surgery through the vaginal approach; and to review the medical literature relating to the anatomy of the retroperitoneal space and the aetiology, diagnosis, management and prognosis of postoperative retroperitoneal haematoma in gynaecology.

Materials and methods: Case presentation of a patient with Stage II vaginal prolapse, who was undergone for vaginal hysterectomy with anterior and posterior vaginal wall repair. She had a retroperitoneal haematoma complication. The patien underwent surgery at Samaritana University Hospital, a level III public hospital and referral centre for the Cundinamarca region, located in Bogotá (Colombia). A search in the literature was conducted in the Medline database through PubMed, Jstor and Lilacs using the MeSH terms "vaginal hysterectomy" and "retroperitoneal haematoma". The search was limited to publications in English and Spanish between 1980 and 2015.

Results: Overall, 15 articles were found, 3 of which describe cases secondary to gynaecological procedures. The final review consisted of 4 topic reviews, 8 case reports 2 case series and 1 observational descriptive study.

Conclusion: Retroperitoneal haematoma is a rare clinical finding and diagnosis requires a high level of clinical suspicion. Several diagnostic tools are available, computed tomography being the most useful. Management has to be individualized in each case. If the patient is stable, the treatments of choice include conservative management, which can be successful, or endovascular interventions. However, in haemodynamically unstable patients, laparotomy is the most recommended treatment approach.

Key words: Retroperitoneal, haematoma, vaginal hysterectomy, haemorrhage.

\section{INTRODUCCIÓN}

El prolapso de órganos pélvicos es uno de los principales desórdenes del piso pélvico en las mujeres, asociado a etiología multifactorial y que incluye varios factores de riesgo conocidos (paridad, edad avanzada, obesidad, anormalidades del tejido conectivo) (1). En Estados Unidos, cerca del $41 \%$ de mujeres entre los 50 a 79 años presentan esta patología, y aproximadamente 200.000 mujeres son intervenidas anualmente para la corrección del prolapso y hasta un $29 \%$ de estas pacientes requerirán reintervención quirúrgica por prolapso recurrente (1). Para Colombia, se cuenta con pocos datos epidemiológicos globales sobre la prevalencia del prolapso; Gutiérrez et al. (2) informan una prevalencia de $47 \%$ de prolapso del componente anterior, y del $32 \%$ del componente posterior en una población estudiada de 1266 pacientes. Este panorama convierte a la histerectomía en una de las cirugías ginecológicas más frecuentes e importantes, siendo el abordaje vaginal el de elección, siempre que no esté contraindicado (35\% de las histerectomías), y más que obvia en casos de prolapso vaginal, asociado a menor morbilidad, periodo de recuperación y estancia hospitalaria (3). Para considerar la vía vaginal se deben tener en cuenta factores como el tamaño uterino, la patología con compromiso extrauterino, la severidad de la patología y la experiencia por parte del cirujano, con el fin de disminuir las complicaciones (3).

Las complicaciones pueden ser intraoperatorias (hemorragia del sitio quirúrgico, lesión vesical, lesión ureteral, lesión intestinal) o complicaciones posquirúrgicas (dolor, hematoma de la cúpula que puede extenderse al retroperitoneo, infección del sitio quirúrgico,) que pueden llevar incluso a reintervención quirúrgica (1).

El hematoma retroperitoneal se caracteriza por el inicio agudo o subagudo de sangrado de los vasos esplácnicos. Cuando se asocia al posoperatorio de histerectomía, se pueden comprometer las ramas de la arteria mesentérica inferior y las ramas de la arteria hipogástrica que sangran al espacio retroperitoneal (4). Existen muy pocas publicaciones sobre el tema en la literatura debido a que es poco frecuente (4). Aunque es una complicación bien reconocida por 
otras especialidades (complicación en cateterización de arteria femoral o en trauma lumbar o pélvico) (4), es una rara condición en cirugía ginecológica. Los reportes más comunes para la especialidad derivan de casos en obstetricia (5). Por esta razón, la incidencia del hematoma retroperitoneal en ginecología es desconocida (4). El diagnóstico muchas veces es tardío porque la presentación es inespecífica y no se encuentran signos y síntomas característicos (4). Sin embargo, la literatura también sugiere que el cirujano ginecólogo debe estar atento a la presentación de esta complicación hemorrágica "oculta" (6), razón por la cual presentamos este caso con el objetivo de hacer una revisión sobre la anatomía del espacio retroperitoneal, la etiología, el diagnóstico, el tratamiento y el pronóstico.

\section{PRESENTACIÓN DEL CASO}

Paciente de 62 años, remitida al Hospital Universitario de La Samaritana, institución pública de tercer nivel de complejidad, centro de referencia de Cundinamarca, ubicado en Bogotá (Colombia), que atiende pacientes afiliados al régimen de seguridad pública, con cuadro clínico de dos años de evolución con sensación de masa en región perineal, asociada a dolor pélvico ocasional y síntomas de incontinencia urinaria de esfuerzo. Como antecedentes personales la paciente tenía osteopenia, con manejo farmacológico con calcio. Antecedentes ginecológicos: G6P5A1V5, última menstruación a la edad de 52 años, última citología en 2011, negativa para malignidad; sin antecedentes quirúrgicos. $\mathrm{Al}$ examen físico se documentó la presencia de cistocele grado III, histerocele grado II y rectocele grado I, sin evidencia de incontinencia con maniobras de valsalva. $\mathrm{Al}$ tacto vaginal presentaba útero pequeño, atrófico, sin masas pélvicas ni otros hallazgos. Fue llevada a junta quirúrgica con estudios complementarios de ecografía transvaginal y urodinamia normales. Se consideró candidata para una histerectomía vaginal con colporrafia anterior y posterior. Previo al procedimiento fue valorada por el servicio de anestesia con los siguientes paraclínicos: hemoglobina (16 g), hematocrito (37\%), plaquetas $(216.000 \mathrm{~mm})^{3}$, tiempos de coagulación (tiempo de protrombina, 11,5; tiempo parcial de tromboplastina, 25), placa de tórax sin alteraciones y electrocardiograma con trastorno inespecífico de la repolarización en cara anterior. En anestesia se clasificó a la paciente con riesgo quirúrgico clase ASA 2, y se reservaron 2 unidades de concentrado globular. Se realizaron los tres procedimientos quirúrgicos: histerectomía vaginal, colporrafia anterior y posterior por técnica habitual. Como hallazgos intraoperatorios, se describió el mismo grado de prolapso, útero atrófico y sangrado estimado en $1000 \mathrm{ml}$. No se presentó ninguna complicación intraoperatoria.

Tres horas después del procedimiento quirúrgico, la paciente presentó hipotensión arterial con cifras de $80 / 40 \mathrm{~mm} / \mathrm{Hg}$ y taquicardia con frecuencia cardiaca 98 latidos por minuto (lpm). Se inició manejo con líquidos endovenosos y se realizó un hemograma que mostró hemoglobina en 9,8 g/dL y hematocrito en 30,5\%. Se realizó el diagnóstico de choque hemorrágico grado II, por lo cual al manejo ya instaurado de líquidos endovenosos se adicionó oxígeno por cánula nasal (2 L/min) y se ordenó transfundir 2 U de glóbulos rojos logrando mejoría en los signos vitales a las 6 horas, con tensión arterial en 106/57 mm/Hg, pero persistió con tendencia a la taquicardia dada por FC 95 lpm. Se realizó hemograma de control posterior a la transfusión con reporte de hemoglobina de 10,2 g/dL y hematocrito en $32,4 \%$. Pese a la aparente respuesta satisfactoria inicial del manejo del choque descrito, a las 26 horas del posquirúrgico presentó nuevamente alteración en los signos vitales con tensión arterial en 79/36 $\mathrm{mm} / \mathrm{Hg}$ y frecuencia cardiaca en $96 \mathrm{lpm}$. En ese momento, la paciente refirió leve dolor abdominal sin otros síntomas adicionales. Al examen físico no se documentaron alteraciones en el abdomen, solo leve dolor a la palpación generalizada; a nivel genitourinario se encontró escaso sangrado vaginal. Se definió nuevamente reanimación hídrica y se solicitaron nuevos paraclínicos de control. A las tres horas de este manejo la paciente presentó aumento del 
dolor abdominal, palidez mucocutánea generalizada, disnea y desaturación a $80 \%$. Pese a que respondió parcialmente al manejo con volumen logrando tensión arterial media mayor a $70 \mathrm{~mm} / \mathrm{Hg}$, la paciente continuó con aumento de la disnea y manifestó dolor torácico. Como primera posibilidad diagnostica se consideró tromboembolismo pulmonar (TEP) frente a sangrado abdominal posoperatorio por descartar. Se solicitaron laboratorios complementarios: función renal, ecografía abdominal, enzimas cardiacas, electrocardiograma, placa de tórax y valoración por el servicio de Medicina Interna. Los resultados se reportaron así: troponina I 0,07 ng/ml; creatinquinasa total, 54,8 U/L; creatinquinasa fracción, MB 11,2 U/L; creatinina, 0,69 mg/dL; nitrógeno ureico, $16,3 \mathrm{mg} / \mathrm{dL}$; hemoglobina, 9,9 g/dL; hematocrito, 29,2\%; plaquetas, $136.000 \mathrm{~mm}^{3}$. Electrocardiograma: ritmo sinusal sin trastornos de la repolarización, ni cambios en el segmento ST.

Con los resultados obtenidos fue valorada por Medicina Interna, servicio que consideró que la paciente presentaba probabilidad intermedia para TEP, por lo cual se inició anticoagulación plena con heparina de bajo peso molecular (enoxaparina $60 \mathrm{mg}$ cada 12 horas) hasta confirmar diagnóstico por angiotac. Seis horas después, la paciente presentó dolor abdominal severo, y al examen físico persistió con taquicardia FC 95 lpm y se documentó distensión abdominal leve, con signos de irritación peritoneal, sin sangrado vaginal. Se revisó ecografía abdominal que reportó presencia de líquido particulado en cavidad abdominal (gotera parietocólica izquierda, polo inferior del lóbulo hepático derecho y líquido periesplénico). Con estos hallazgos al examen físico, sumados al reporte de la ecografía y el descenso en el nivel de hemoglobina, se diagnosticó sangrado intraabdominal, por lo cual se programó para laparotomía exploratoria urgente. A las 48 horas después del procedimiento quirúrgico inicial se realizó laparotomía con los siguientes hallazgos: hemoperitoneo oscuro cuantificado en 2000 cc, cúpula vaginal y pedículos vasculares sin evidencia de sangrado activo, pero se observó hematoma contenido que se extendía al espacio retroperitoneal involucrando la cara posterior del lecho vesical y paredes laterales de la pelvis, sin que se decidiera realizar exploración del mismo. Se llevó a cabo lavado de la cavidad abdominal verificando la hemostasia. El procedimiento finalizó sin complicaciones. Se trasladó la paciente a recuperación para continuar vigilancia del posquirúrgico inmediato durante el cual se hizo nuevamente transfusión de 2 U de concentrado globular y 10 U de plasma; se consideró cubrimiento antibiótico con clindamicina $600 \mathrm{mg}$ cada 8 horas y gentamicina $240 \mathrm{mg}$ cada día, y se solicitó control de paraclínicos que documentaron hemoglobina en 10,5 g y hematocrito de 31 \%. La paciente presentó evolución clínica hacia la mejoría, por lo cual 12 horas después de la última intervención quirúrgica se reinició profilaxis antitrombótica. Se tomó angiotac y se descartó TEP.

La paciente completó tres días de cubrimiento antibiótico intrahospitalario y por evolución satisfactoria con último reporte de hemoglobina en 11,8 g, se dio egreso. Se realizó control posquirúrgico ambulatorio a los 8 y 15 días, y la paciente se encontraba asintomática, sin dolor, con signos vitales normales y evolución satisfactoria, por lo cual se finalizó el seguimiento.

Aspectos éticos. Se solicitó autorización por escrito a la paciente y al Hospital Universitario de La Samaritana para publicar el caso. Se garantizó la confidencialidad de la información protegiendo los derechos de la paciente.

\section{MATERIALES Y MÉTODOS}

Se realizó una búsqueda de literatura médica en bases de datos disponibles en Internet: Medline vía PubMed, Jstor y Lilacs, con la terminología MeSH "hysterectomy vaginal" y "retroperitoneal hematoma”. La búsqueda se limitó a los idiomas inglés y español entre los años 1980 a 2015.

\section{RESULTADOS}

Se encontraron 15 artículos de utilidad para el caso en particular, de los cuales 3 describen casos 
secundarios a procedimientos ginecológicos. La revisión final se conforma de: 4 revisiones de tema, 8 reportes de caso, 2 series de casos, 1 estudio observacional descriptivo.

Anatomía. El espacio retroperitoneal es una región rica en estructuras vitales y es un sitio de hemorragia mayor por ser muy vascularizado (7). Anatómicamente, el retroperitoneo se divide en 3 zonas: zona I o central (área medial al hilio renal), contiene la aorta abdominal, la vena cava inferior, el tronco celiaco, la arteria mesentérica superior y la vasculatura proximal renal. Zona II o lateral, incluye las glándulas suprarrenales, los riñones y el tracto genitourinario proximal. Finalmente, la zona III o pélvica, que contiene el recto, los vasos iliacos y sus ramas (7).

Etiología. Etiológicamente, la hemorragia retroperitoneal puede clasificarse en espontánea o secundaria. Entre sus causas espontáneas se encuentran alteraciones de la coagulación o uso de anticoagulantes, edad avanzada, hipertensión, vasculitis o ruptura de aneurismas, tumores benignos o malignos y embarazo. Como causas secundarias están el trauma o la cirugía abdominal o pélvica reciente (7) y cirugía urológica o litotripsia interna (8).

En cuanto a procedimientos ginecológicos se encontraron solo tres reportes de caso que informaron esta complicación: el primero, publicado en 1986 como una complicación posterior a la realización de un cono frío del cérvix (9); el segundo, publicado en 1987, similar al caso que reportamos posterior a una histerectomía vaginal (10), y el último, en 2007, después de una corrección de prolapso vaginal con malla (6). La mayoría de casos en ginecología y obstetricia, y de los cuales incluso se encuentran algunas revisiones, están asociados al parto vaginal con una incidencia de 1/4000 partos (4).

Diagnóstico. El cuadro clínico es inespecífico, lo que a menudo conlleva un diagnóstico tardío (8), a excepción de los casos en los que se manifiesta rápidamente con inestabilidad hemodinámica, lo cual ocurre con mayor frecuencia posterior a trauma (7). El porcentaje de pacientes sintomáticos es de aproximadamente $30 \%$, y debido a la naturaleza del hematoma de autolimitarse o de no aumentar significativamente de tamaño, se documentan menos síntomas (8). En una serie de 26 casos, los síntomas más comunes descritos fueron: dolor de intensidad variable a nivel lumbar (46\%) o de flancos (23\%), equimosis como los signos de Gray Turner y Cullen, eventualmente taquicardia inexplicada (31\%), hipotensión (92\%) y descenso en los niveles de hemoglobina y hematocrito (58\%) debido a la pérdida de grandes volúmenes sanguíneos (11).

En cuanto a las imágenes diagnósticas: la radiografía simple de abdomen permite sospechar el hematoma retroperitoneal ante la pérdida de la sombra del psoas (12). La ecografía es útil para identificar líquido libre en cavidad de tipo particulado y masa retroperitoneal mal definida; sin embargo, requiere de un entrenamiento en identificación del hematoma y presenta limitaciones por el gas, tejido adiposo, dolor y colocación del transductor (13). Actualmente, la tomografía axial computarizada (TAC) es el estudio de elección para el diagnóstico de hematoma retroperitoneal ya que proporciona información con respecto a la localización y a la extensión de la colección (8). El hematoma se puede sospechar ante la presencia de una imagen de densidad de tejido blando anormal. La tomografía helicoidal permite una mayor calidad de imágenes mediante reconstrucción multiplanar y una máxima intensidad de su proyección, reduciendo artefactos (12). Finalmente, la resonancia magnética no parece aportar ventajas sobre la TAC, por lo cual solo se realiza en casos en los cuales esta última no es concluyente (8).

Tratamiento. La realización de manejo quirúrgico o conservador depende de la etiología, el tamaño y la extensión del hematoma asociado al estado hemodinámico de la paciente, compresión de estructuras vecinas y el riesgo de hemorragia retroperitoneal masiva (4). En pacientes estables hemodinámicamente, el manejo conservador puede ser igualmente exitoso y se basa en la reposición de la volemia, corrección de los trastornos de la coagu- 
lación, terapia transfusional y medidas de soporte, por lo general bajo monitorización en unidades de alta dependencia o de cuidado intensivo (UCI) (4).

Es por esto que en varios casos reportados (8), incluido el presente, la evidencia de inestabilidad hemodinámica refractaria al tratamiento médico constituyó la indicación de laparotomía. El objetivo del procedimiento sería controlar el sangrado activo y remover el hematoma (14). Sin embargo, se debe aclarar que remover el hematoma puede llevar a un peor resultado debido a una hemorragia masiva al romper el efecto mecánico del taponamiento del retroperitoneo (4), por lo cual se recomienda dejar empaquetado y reexplorarse en 24 a 48 horas (15). La tendencia actual en pacientes estables es el manejo por técnicas endovasculares como una alternativa a la cirugía (14).

En instituciones donde se cuente con el recurso humano y tecnológico, las principales opciones de manejo son: el embolismo selectivo intraarterial (utilizando coils, gelatina o alcohol polivinílico) o la colocación de endoprotesis (stents) (14).

Pronóstico. Depende fundamentalmente de la velocidad y volumen de la hemorragia y del diagnóstico oportuno (8). En la evolución de la complicación se encuentran dos escenarios: la corrección autolimitada del sangrado hasta el choque hemorrágico, que se presenta en el $40 \%$ de los casos, alcanzando una alta mortalidad: hasta el $25 \%$ (7). En este caso en particular, el diagnóstico fue tardío (48 horas posterior a la cirugía inicial) debido a lo inespecífico de los síntomas. Sin embargo, una vez confirmado el diagnóstico intraoperatorio, la paciente respondió favorablemente al manejo de no exploración del hematoma.

\section{CONCLUSIÓN}

El hematoma retroperitoneal es una rara entidad que requiere de un alto índice de sospecha clínica para su diagnóstico. El avance en las intervenciones menos invasivas permite tener varias opciones de manejo, disminuyendo la morbimortalidad de la cirugía abierta. Sin embargo, en pacientes inestables hemodinámicamente, la laparotomía es el manejo de elección con el objetivo de controlar el sangrado. Se recomienda no manipular el hematoma debido al riesgo de desencadenar una hemorragia masiva. Se requiere de la participación multidisciplinaria durante el seguimiento posquirúrgico (8).

\section{REFERENCIAS}

1. Siddiqui NY, Edenfield AL. Clinical challenges in the management of vaginal prolapse. Int J Women's Health. 2014;2:83-94.

2. Gutiérrez AF, Solórzano MC, Trujillo CG, Plata M. Prevalencia de prolapso de piso pélvico en pacientes con incontinencia urinaria de esfuerzo. Urol Colomb. 2012;21:41-9.

3. Salinas H, Pasten J, Naranjo B, Carmona S, Retamales M, Díaz G, et al. Análisis económico de la histerectomía abdominal versus vaginal en el hospital clínico de la Universidad de Chile. Revision de 2338 casos. Rev Chil Obstet Ginecol. 2006;71:227-33.

4. Rafi J, Muppala H. Retroperitoneal haematomas in obstetrics: literature review. Arch Gynecol Obstet. 2010;281:435-41.

5. El-agwany AS. Retzius space haematoma with retroperitoneal dissection after spontaneous vaignal delivery: an unusual presentation of ruptured paravesical plexus managed by bilateral internal iliac artery ligation. Polish Annals of medicine. 2015 (article in press).

6. Gangam N, Kanee A. Retroperitoneal hemorrhage after a vaginal mesh prolapse procedure. Obstet Gynecol. 2007;110:463-4.

7. Kasotakis G. Retroperitoneal and rectus sheath hematomas. Surg Clin North Am. 2014; 94:71-6.

8. Suárez G, Valera Z, Gómez MA, Docobo F, Álamos JM. Etiología y diagnóstico del hematoma retroperitoneal complicado, actitud e indicación quirúrgica. Cir Esp. 2005; 78:328-30.

9. Brown CF, Mashini IS, Turner WA, Gallup DG. Retroperitoneal hematoma: an unusual complication of cold knife conization of the cervix. Obstet Gynecol. 1986;68:66S-7.

10. Lev-Gur M, Patel S, Greston WM, McGill F. Pararenal hematoma as a complication of vaginal hysterectomy. A case report. J Reprod Med. 1987;32:68-71.

11. Farouque HM, Tremmel JA, Raissi S. Risk factors for the development of retroperitoneal hematoma after 
percutaneous coronary intervention in the era of glycoprotein IIb/IIIa inhibitors and vascular closure devices. J Am Coll Cardiol. 2005;45:363-8.

12. Lindner A, Zierz S. Images in clinical medicine. Retroperitoneal hemorrhage. N Engl J Med. 2001; 344:348.

13. Ruchholtz S, Waydhas C, Lewan U, Pehle B, Taeger G, Kühne C, et al. Free abdominal fluid on ultrasound in unstable pelvic ring fracture: is laparotomy always necessary? J Trauma. 2004;57:285-7.
14. Chan YC, Morales JP, Reidy JF, Taylor PR. Management of spontaneous and iatrogenic retroperitoneal haemorrhage: conservative management, endovascular intervention or open surgery? Int J Clin Pract. 2008;62:1604-13.

15. Dae-Yeon W, Sang-Dong K, Sun-Chul P, In-Sung M, Ji K. abdominal compartment syndrome due to spontaneous retroperitoneal hemorrhage in a patient undergoing anticoagulation. Yonsei Med J. 2011;52:358-61.

\section{Conflicto de intereses: ninguno declarado.}

